\title{
Avaliação da inclinação do incisivo inferior através da tomografia computadorizada
}

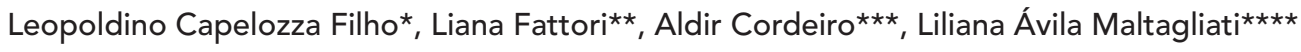

\section{Resumo}

Introdução: durante muito tempo, a Ortodontia baseou-se em valores normativos obtidos através das análises cefalométricas existentes, utilizando a telerradiografia em norma lateral como exame principal no diagnóstico ortodôntico, ditando o plano de tratamento. $\mathrm{O}$ incisivo inferior, em particular, recebeu normas cefalométricas propostas por diversos autores, dentre eles Tweed. Entretanto, os exames radiográficos possuem limitações, pois, em uma imagem bidimensional de uma estrutura tridimensional, a superposição de determinadas estruturas ósseas e dentárias é constante. O advento da tomografia computadorizada disponibilizou meios diagnósticos mais precisos e confiáveis, principalmente pela possibilidade de obtenção da imagem das estruturas em três dimensões. Nesse contexto, parece razoável questionar os dados oriundos da análise clássica da radiografia lateral da face, com intuito de criar fatores de comparação com dados que passarão a ser gerados a partir da mesma imagem obtida pela tomografia. Objetivos: para isso, propusemo-nos a avaliar o ângulo formado entre o longo eixo do incisivo inferior e o plano mandibular, a grandeza IMPA da cefalometria, na telerradiografia convencional e na tomografia computadorizada. Métodos: dezenove pacientes selecionados para tratamento ortodôntico constituíram a amostra deste estudo, 12 do gênero feminino e 7 do masculino, com idades entre 16 anos e 4 meses e 28 anos e 2 meses. Após a coleta de dados, feita por dois examinadores, análises estatísticas para a obtenção dos erros intra e interexaminadores foram feitas, utilizando nível de significância de 5\%. Resultados e Conclusões: concluiu-se que a obtenção dos valores para o ângulo formado entre o longo eixo do incisivo inferior e o plano mandibular (IMPA) na telerradiografia lateral é um método confiável para uso clínico, porque embora apresente, como regra, valores menores que os encontrados em tomografia computadorizada, as diferenças não são clinicamente significativas.

Palavras-chave: Cefalometria. Tomografia computadorizada. Ortodontia.

* Professor doutor da Faculdade de Odontologia da Universidade de São Paulo, USP-Bauru. Membro do setor de Ortodontia do HRAC da Universidade de São Paulo, USP-Bauru. Professor do programa de pós-graduação em Ortodontia da Faculdade de Odontologia de Araçatuba-UNESP.

** Mestre em Ortodontia pela UMESP. Especialista em OFM e Ortodontia pelo CFO.

$\star \star \star$ Especialista em Ortodontia-PROFIS.

**** Professora doutora em Ortodontia pela FOB-USP. Professora do programa de pós-graduação em Odontologia (Mestrado) - área de concentração Ortodontia da UMESP e coordenadora do curso de especialização em Ortodontia da UMESP. 


\section{INTRODUÇÃO}

Durante muito tempo, a Ortodontia utilizou a telerradiografia em norma lateral como exame principal no diagnóstico ortodôntico, baseada em valores normativos obtidos nas análises cefalométricas existentes, que ditavam o plano de tratamento a ser seguido. Com os avanços na área de diagnóstico - dentre eles a utilização da análise facial e a individualização dos resultados apresentados nos exames radiográficos - desenvolveu-se a consciência das limitações da cefalometria, determinando aos ortodontistas uma utilização mais racional desse método de análise na elaboração do diagnóstico.

Atualmente, a cefalometria, como qualquer exame complementar, auxilia o profissional no estabelecimento do plano de tratamento, em conjunto com outros dados como a anamnese, análise facial e análise de modelos. Além disso, é também empregada no planejamento de casos que necessitam de cirurgia ortognática, na avaliação do crescimento craniofacial e dos resultados obtidos pelas terapias ortodônticas, além do uso em pesquisas científicas para a análise das mais variadas estruturas e medidas como, por exemplo, a relação que os dentes anteriores, sobretudo os incisivos, mantêm com suas estruturas de suporte.

Apesar de Angle (apud FINLAY ${ }^{5}$, 1980) afirmar que qualquer ortodontista seria capaz de classificar uma má oclusão apenas pela face, um parâmetro que não fosse puramente estético precisava ser adotado. A premissa adotada foi a de que o ideal dentário e, porque não, estético, seria alcançado quando os incisivos inferiores fossem posicionados verticalmente sobre sua base óssea e todos os demais dentes organizados a partir deste parâmetro. Assim sendo, a meta terapêutica passou a ser a verticalização dos incisivos inferiores com uma variação mínima de inclinação em relação ao plano mandibular ${ }^{21}$. Para indivíduos brasileiros com oclusão normal natural, o valor médio de IMPA encontrado foi de $93,85^{\circ}$, com variação de $84^{\circ}$ a $105,5^{\circ}$, segundo Martins ${ }^{16}$.

Tweed $^{21}$, em 1954, relacionou o incisivo inferior com o plano de Frankfurt, criando um tri- ângulo formado pelo plano de Frankfurt, o plano mandibular e o longo eixo do incisivo inferior. Com conhecimento de trigonometria, que estabelece que a soma dos ângulos internos de um triângulo resulta, invariavelmente, em $180^{\circ}$, Tweed desenvolveu sua análise, que obtém o valor ideal do posicionamento do incisivo inferior (IMPA Incisor Mandibular Plane Angle) a partir do valor encontrado nos outros ângulos (FMA - Frankfurt Mandibular Plane Angle e FMIA - Frankfurt Mandibular Incisor Angle).

Em 1948, Downs ${ }^{4}$ analisou a inclinação do incisivo inferior de indivíduos com oclusão normal e que nunca haviam sido submetidos ao tratamento ortodôntico. A inclinação do incisivo inferior foi determinada pela intersecção do longo eixo do incisivo inferior com o plano palatino e o valor normativo estabelecido foi de $104,5^{\circ}$.

Várias são as análises cefalométricas ${ }^{20}$ disponíveis com o intuito de auxiliar o diagnóstico ortodôntico, porém, estas devem ser usadas com parcimônia, por dois motivos: não levam em conta as características individuais dos pacientes, utilizando médias para a definição do diagnóstico ortodôntico, e possuem erros intrínsecos do método. Estudos utilizando telerradiografias em norma lateral provaram que a identificação dos pontos cefalométricos das diversas análises existentes é a maior fonte dos erros ${ }^{1}$.

Entretanto, os exames radiográficos, incluindo as telerradiografias, como anteriormente mencionado, possuem limitações, pois, em uma imagem bidimensional de uma estrutura tridimensional, a sobreposição de determinadas estruturas ósseas e dentárias é uma constante.

Isto acontece claramente na avaliação do posicionamento dos incisivos inferiores, que estão localizados na região anterior da mandíbula, área de difícil visualização, pois nesta região ocorre uma sobreposição das coroas e raízes dentárias de incisivos e caninos, dificultando o correto traçado da raiz do incisivo mais vestibularizado e, conseqüentemente, a correta leitura de sua inclinação. Isso é, 
provavelmente, ainda mais intenso na presença de más oclusões, onde é freqüente o apinhamento dos dentes anteriores inferiores. Provavelmente, a real posição de um incisivo inferior na sínfise mandibular nunca é visível, mas sempre inferida através de pistas morfológicas que a imagem radiográfica permite vislumbrar.

$\mathrm{O}$ avanço nos exames por imagem, como a utilização da tomografia computadorizada, disponibiliza meios para diagnósticos mais precisos e com grande confiabilidade, principalmente por possibilitar a obtenção das estruturas em três dimensões $^{8,9}$. A fabricação de um tomógrafo computadorizado volumétrico específico para a região bucomaxilofacial ${ }^{6,7}$ - com utilização de menor dose de radiação ${ }^{13,17,18,19}$ - aliada a um software que permite a realização de mensurações nos cortes tomográficos realizados ${ }^{3}$, fez surgir a possibilidade de avaliar a confiabilidade das medidas cefalométricas existentes. Para desenvolver uma metodologia para essa avaliação, foi escolhida a grandeza IMPA. Admite-se que, pela representação fiel das estruturas necessárias para se chegar a seu valor no exame tomográfico, foi preciso também avaliar o que se obtém com a avaliação clássica por meio do cefalograma obtido a partir da telerradiografia em norma lateral.

\section{MATERIAL E MÉTODOS}

A amostra utilizada neste estudo foi constituída por 19 indivíduos, sendo 12 do gênero feminino e 7 do masculino, com idades entre 16 anos e 4 meses e 28 anos e 2 meses, selecionados para tratamento ortodôntico na clínica do departamento de pós-graduação em Odontologia, área de concentração Ortodontia, da Universidade Metodista de São Paulo. Obedeceu-se os seguintes critérios de inclusão: apresentar dentadura permanente e não ter ausências dentárias na região anterior. Além da telerradiografia lateral, foram realizados e utilizados exames de tomografia computadorizada volumétrica, para a realização das mensurações propostas.

\section{Obtenção dos exames}

Telerradiografia lateral

A telerradiografia lateral foi obtida no setor de Radiologia do departamento de pós-graduação em Odontologia, área de concentração Ortodontia, da Universidade Metodista de São Paulo, seguindo-se o protocolo do departamento. Todas as radiografias foram realizadas pelo mesmo operador.

\section{Tomografia computadorizada}

Os exames tomográficos volumétricos foram realizados no Centro de Radiologia da Associação Paulista dos Cirurgiões-Dentistas (APCD - Central, São Paulo), sob a supervisão do radiologista responsável, utilizando o tomógrafo computadorizado volumétrico NewTom modelo DVT-9000 (NIM - Verona - Itália). Para a reformatação das imagens e mensuração dos valores de IMPA, foi empregado o software QR-DVT 9000 (NIM - Verona - Itália).

Durante a realização do exame, o paciente foi colocado em decúbito dorsal, em posição horizontal na mesa auxiliar do tomógrafo, com a cabeça apoiada em um dispositivo que auxilia seu correto posicionamento, ou seja, alinhada ao corpo, sem qualquer rotação. A cabeça do paciente foi, então, posicionada no gantry, que é a região central do tomógrafo, onde incide o feixe cônico de raios $\mathrm{X}$, para a aquisição da anatomia digital do paciente. Para o correto posicionamento da cabeça, o equipamento possui dois feixes de laser, o primeiro deve coincidir com a linha média da face e o segundo incidir na região temporal do crânio. Em seguida, o aparelho faz um único escaneamento da região, com um giro de $360^{\circ}$ do tubo de raios $\mathrm{X}$ e de seu sensor ao redor da cabeça do paciente e, após 72 segundos de escaneamento, uma imagem digital em volume da região é adquirida.

O tomógrafo computadorizado NewTom 9000 foi desenvolvido especialmente para a região bucomaxilofacial, proporcionando imagens volumétricas, como os tomógrafos médicos, porém com uma significante redução da radiação a que o pa- 
ciente é exposto, com as seguintes vantagens: excelente imagem, de baixo custo e risco; confiabilidade na reprodução das estruturas digitalizadas, para as medidas lineares ${ }^{12,14}$ e para as angulares ${ }^{14}$; fácil manuseio e, ainda, tempo de exame reduzi$\mathrm{do}^{8}$. Além disso, apenas uma sessão para aquisição da imagem tomográfica em volume permite que sejam feitas reconstruções nos mais diversos planos, com o auxílio do software, proporcionando múltiplas imagens, como, por exemplo: o scout lateral e frontal, imagens semelhantes às telerradiografias lateral e frontal; a reconstrução oclusal; panorâmicas; reconstruções transaxiais; coronais, além das reconstruções em $3 \mathrm{D}^{2,17,18}$.

\section{Mensuração do IMPA}

Na telerradiografia lateral

Previamente aos procedimentos de obtenção do valor do IMPA, dois examinadores ortodontistas foram calibrados para mensuração na telerradiografia.

\section{Na tomografia computadorizada}

Após a aquisição das imagens, ambos examinadores fizeram a mensuração do valor do IMPA, com auxílio do software QR DVT-9000. Para cada exame feito, os procedimentos abaixo foram repetidos.

A partir do scout lateral (Fig. 2), que é a imagem em duas dimensões do exame, fez-se a reconstrução primária das imagens para obtenção dos slices dentro dos parâmetros desejados. Como o plano de referência para a obtenção do valor do IMPA é o plano mandibular, utilizou-se este plano como referência para a reconstrução primária da mandíbula, de modo que todos os slices tivessem este plano como referência e fossem paralelos a ele, viabilizando a mensuração do valor de inclinação do incisivo mais vestibularizado (Fig. 3).

Com toda a imagem volumétrica reformatada segundo o plano mandibular, escolheu-se o slice, a imagem axial $(0,3 \mathrm{~mm}$ de espessura $)$ mais próxima ao centro da coroa clínica dos dentes anteriores, de modo que foi possível visualizar qual dos incisivos centrais inferiores estava mais vestibularizado.

A partir desta imagem axial selecionada, foram feitos os procedimentos para a reconstrução secundária da mesma. Desta forma, pode-se ter a visualização de todos os dentes axialmente, como uma radiografia oclusal. Com a utilização

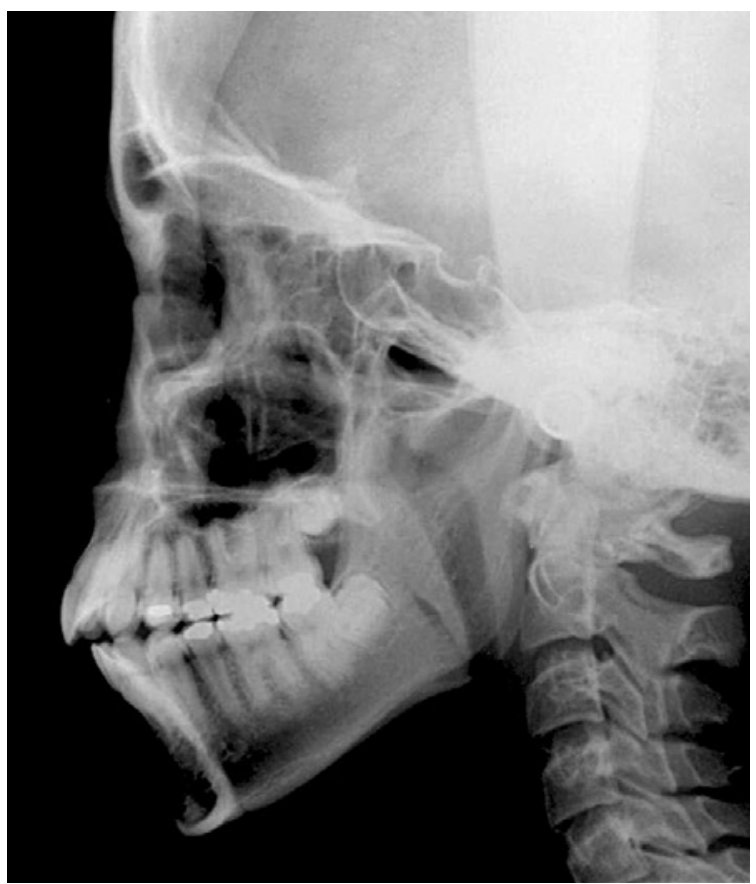

FIGURA 1 - Telerradiografia lateral.

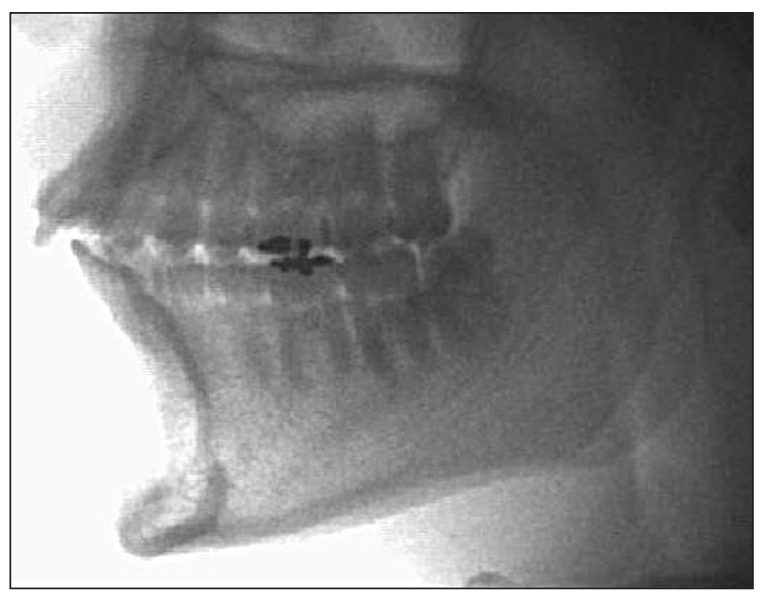

FIGURA 2 - Scout lateral. 


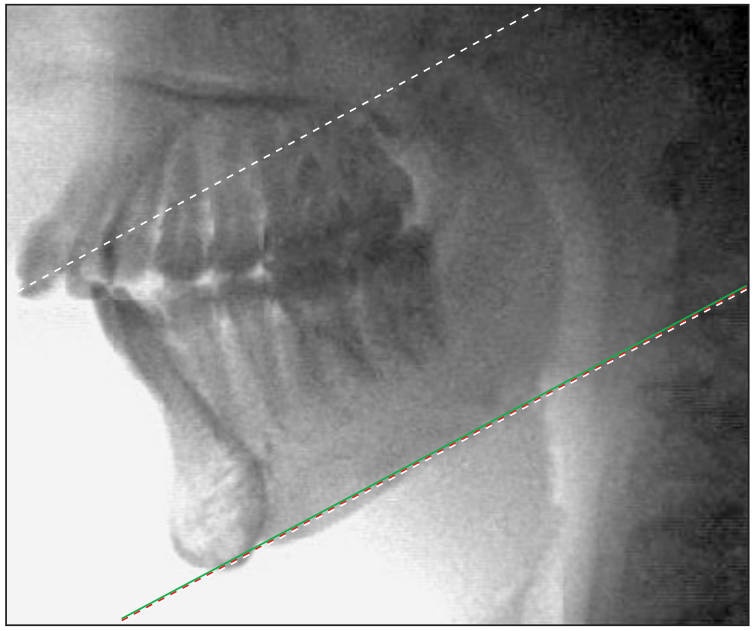

FIGURA 3 - Reformatação primária - plano mandibular.

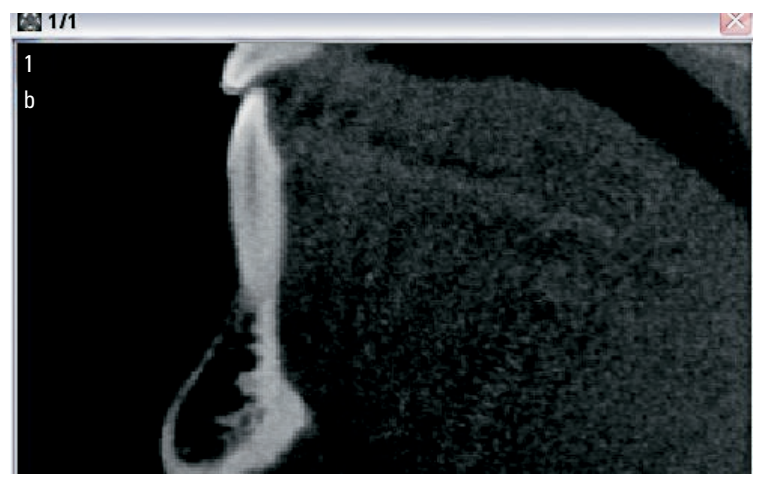

FIGURA 5 - Imagem transaxial.

de ferramentas do software para definir a imagem transaxial do dente selecionado, os procedimentos descritos a seguir foram realizados: construiu-se uma linha tangente à porção mais vestibular da face vestibular do dente, eqüidistante das faces mesial e distal. Sobre esta linha, com a ferramenta de angulação do software, colocou-se o vértice do ângulo sobre o centro da face vestibular e acertouse a angulação em $90^{\circ}$, a fim de se obter a perpendicular a esta tangente vestibular, representando o exato longo eixo do dente (Fig. 4).

Sobre esta linha perpendicular, fez-se a reconstrução secundária da imagem, para obtenção da imagem transaxial do dente (Fig. 5). Na imagem transaxial obtida, com a representação do dente completo, inserido entre as corticais ósseas dentoalveolares e toda a anatomia mandibular da região,

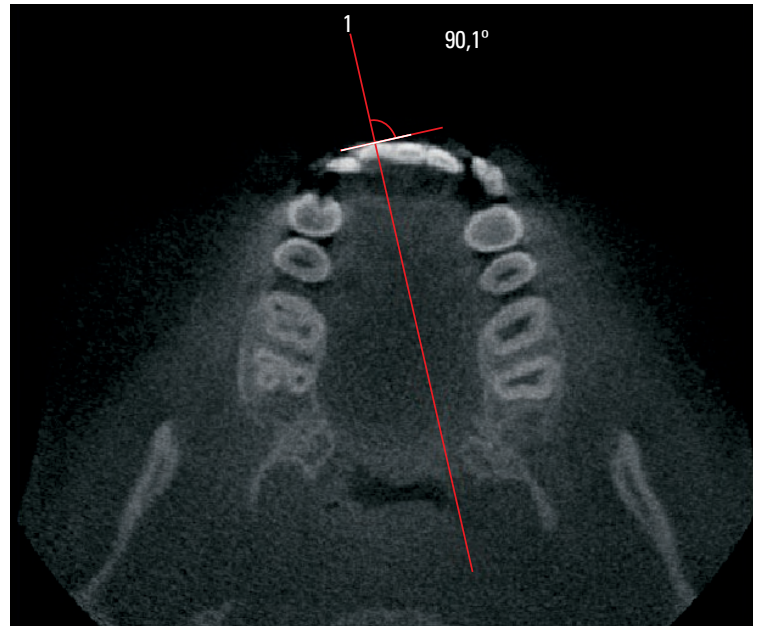

FIGURA 4 - Imagem axial com área para a reconstrução secundária.

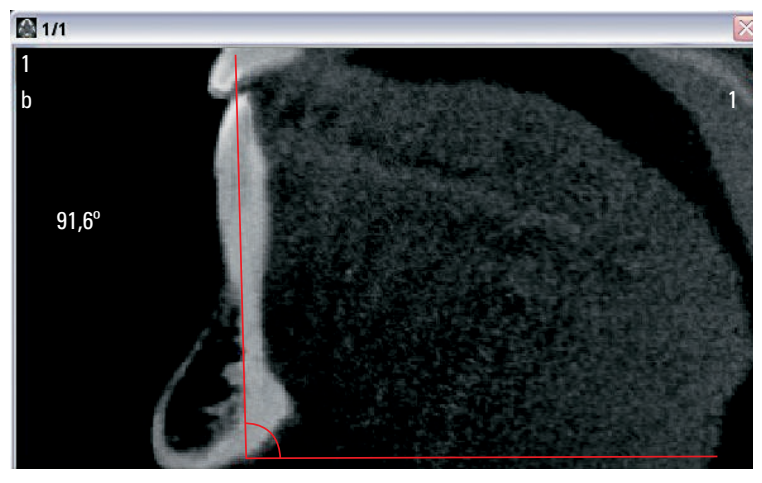

FIGURA 6 - Obtenção do valor do IMPA.

fez-se os procedimentos, com o auxílio das ferramentas do software, para se determinar o valor exato do IMPA, sem sobreposição de qualquer estrutura adjacente, dentária ou óssea.

Com a ferramenta de angulação, obtém-se o valor exato da inclinação do incisivo inferior, pelo ângulo formado entre o longo eixo do dente em questão e o plano mandibular, representado pelo limite da imagem, obtido na reconstrução primária do exame (Fig. 6). O procedimento utilizado foi o seguinte: ajustou-se o vértice do ângulo sobre o limite da imagem transaxial com um de seus braços paralelo ao plano mandibular, representado pelo limite da imagem, e o outro ajustado ao longo eixo do dente, obtendo-se automaticamente o valor da inclinação do incisivo com o plano mandibular (IMPA). 


\section{Avaliação do erro metodológico e análise estatística}

Para o erro intra-examinador, após 20 dias das mensurações terem sido realizadas, foram reavaliados 5 indivíduos escolhidos aleatoriamente, para a determinação do erro sistemático e do erro casual. Este procedimento foi realizado pelos dois pesquisadores. Para o erro sistemático, foi utilizado o teste $t$ pareado (com nível de significância de 5\%) e, para o erro casual, a fórmula de Dahlberg.

Para o erro interexaminador, foi aplicado o teste $t$ de Student, utilizando nível de significância de $5 \%(\mathrm{p}<0,05)$.

Após a execução de todos estes procedimentos, em ambos os exames, os valores do IMPA foram analisados estatisticamente para a verificação do cálculo da média e desvio-padrão, o teste t pareado para as amostras numéricas relacionadas, comparando-se os valores encontrados para o IMPA, na telerradiografia e na tomografia computadorizada.

Foi utilizado o nível de significância descritivo considerando-se como estatisticamente significantes resultados com $\mathrm{p}<0,05$ ou $5 \%$.

\section{RESULTADOS}

Os resultados estão dispostos em tabelas, descrevendo o erro metodológico intra e interexaminadores e a avaliação comparativa entre a mensuração do valor de IMPA pela telerradiografia e tomografia computadorizada.

Tabela 1 - Valores de IMPA, média, desvio-padrão, valor máximo e mínimo obtidos na TC para as duas mensurações, dos dois examinadores, na análise de erro metodológico.

\begin{tabular}{|c|c|c|c|c|}
\hline & \multicolumn{2}{|c|}{ examinador 1} & \multicolumn{2}{|c|}{ examinador 2} \\
\hline & $1^{\mathrm{a}}$ mensuração & $2^{\mathrm{a}}$ mensuração & $1^{\mathrm{a}}$ mensuração & $2^{\mathrm{a}}$ mensuração \\
\hline 1 & 103,5 & 103,9 & 102,5 & 103,2 \\
\hline 2 & 89,7 & 88 & 89,3 & 90,3 \\
\hline 3 & 99,9 & 98,8 & 98,3 & 97,6 \\
\hline 4 & 87,8 & 87,8 & 88,5 & 88,2 \\
\hline 5 & 84 & 83,7 & 84 & 85,3 \\
\hline média & 92,98 & 92,44 & 92,52 & 92,92 \\
\hline d.p. & 8,318 & 8,506 & 7,618 & 7,328 \\
\hline máx. & 103,5 & 103,9 & 102,5 & 103,2 \\
\hline mín. & 84 & 83,7 & 84 & 85,3 \\
\hline
\end{tabular}

Tabela 2 - Valores para o erro sistemático (teste t pareado) e erro casual (Dahlberg) de cada um dos examinadores.

\begin{tabular}{|c|c|c|c|c|c|c|c|c|c|c|c|}
\hline & & \multicolumn{5}{|c|}{ examinador 1} & \multicolumn{5}{|c|}{ examinador 2} \\
\hline & & 1 & 2 & 3 & 4 & 5 & 1 & 2 & 3 & 4 & 5 \\
\hline $\begin{array}{c}\text { diferença } \\
1^{\text {a }} \text { e } 2^{2^{\mathrm{a}}} \text { mensuração }\end{array}$ & & $-0,4$ & 1,7 & 1,1 & 0 & 0,3 & $-0,7$ & -1 & 0,7 & 0,3 & $-1,3$ \\
\hline Dahlberg & & 0,12 & 0,53 & 0,34 & 0,00 & 0,09 & 0,22 & 0,31 & 0,22 & 0,09 & 0,41 \\
\hline \multirow{2}{*}{ teste t pareado } & $t$ & & & 1,420 & & & & & $-1,040$ & & \\
\hline & p & & & 0,229 & & & & & 0,357 & & \\
\hline
\end{tabular}


Tabela 3 - Valores de t e p no teste estatístico entre as medidas dos dois examinadores, na telerradiografia e na TC.

$\begin{array}{ccc} & \text { teste } t & \\ & \text { TC } & \text { tele } \\ \mathbf{t} & 1,484 & 1,138 \\ \mathbf{p} & 0,155 & 0,27\end{array}$

Tabela 5 - Resultados estatísticos entre as mensurações do IMPA na telerradiografia e na TC de cada um dos examinadores.

$\begin{array}{ccc} & \text { teste } \mathrm{t} & \\ & \text { Ex. 1 } & \text { Ex. } 2 \\ \mathbf{t} & 2,725 & 3,244 \\ \mathbf{p} & 0,014 & 0,005\end{array}$

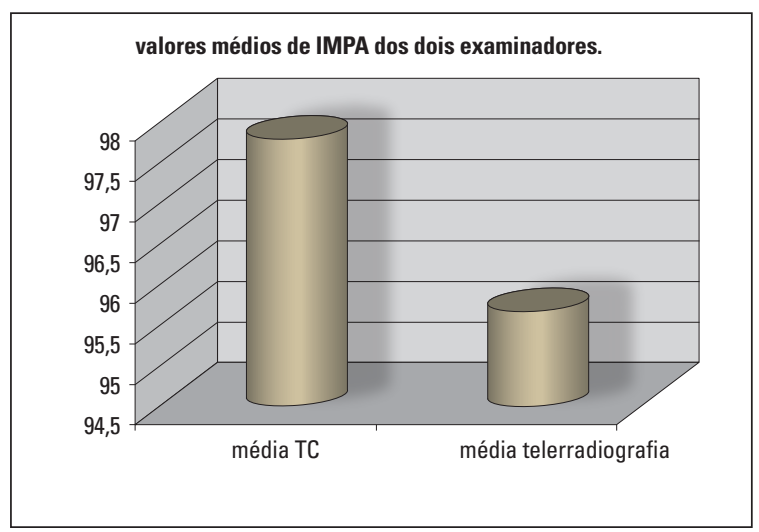

GRÁFICO 1 - Valores médios de IMPA na TC e na telerradiografia, encontrados pelos dois examinadores.

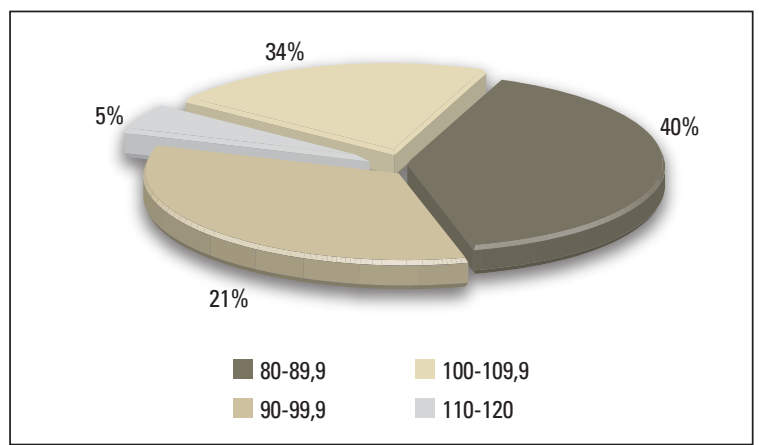

GRÁFICO 2 - Distribuição dos valores de IMPA na TC.
Tabela 4 - Valores angulares encontrados, média, desvio padrão, valor máximo e mínimo do IMPA na amostra, valor de " $t$ " e " $p$ ", examinador 1 e 2.

\begin{tabular}{|c|c|c|c|c|}
\hline & \multicolumn{2}{|c|}{ IMPA TC } & \multicolumn{2}{|c|}{ IMPA Tele } \\
\hline & Ex.1 & Ex.2 & Ex.1 & Ex.2 \\
\hline 1 & 98,5 & 98,3 & 98,5 & 97 \\
\hline 2 & 85,6 & 86,2 & 82 & 84 \\
\hline 3 & 96,2 & 96,6 & 93 & 95 \\
\hline 4 & 103,5 & 102,5 & 99 & 99 \\
\hline 5 & 107,8 & 106,8 & 100 & 97 \\
\hline 6 & 104 & 103,5 & 98,5 & 100 \\
\hline 7 & 89,7 & 89,3 & 91 & 88 \\
\hline 8 & 91,6 & 92 & 89,5 & 91 \\
\hline 9 & 113,9 & 113,3 & 110 & 108 \\
\hline 10 & 99,9 & 98,3 & 98 & 97 \\
\hline 11 & 101,7 & 100,9 & 98 & 101 \\
\hline 12 & 100,5 & 101,4 & 104 & 102 \\
\hline 13 & 87,8 & 88,5 & 90 & 87 \\
\hline 14 & 102,6 & 102,9 & 106 & 107 \\
\hline 15 & 93,3 & 94,6 & 93 & 88 \\
\hline 16 & 99,2 & 97,1 & 99 & 92 \\
\hline 17 & 84 & 84 & 81 & 86 \\
\hline 18 & 99,6 & 98,8 & 97 & 96 \\
\hline 19 & 101,3 & 99,7 & 97 & 95 \\
\hline média & 97,93 & 97,62 & 96,03 & 95,26 \\
\hline d.p. & 7,688 & 7,297 & 7,298 & 6,83 \\
\hline máx. & 113,9 & 113,3 & 110 & 108 \\
\hline mín. & 84 & 84 & 81 & 84 \\
\hline
\end{tabular}

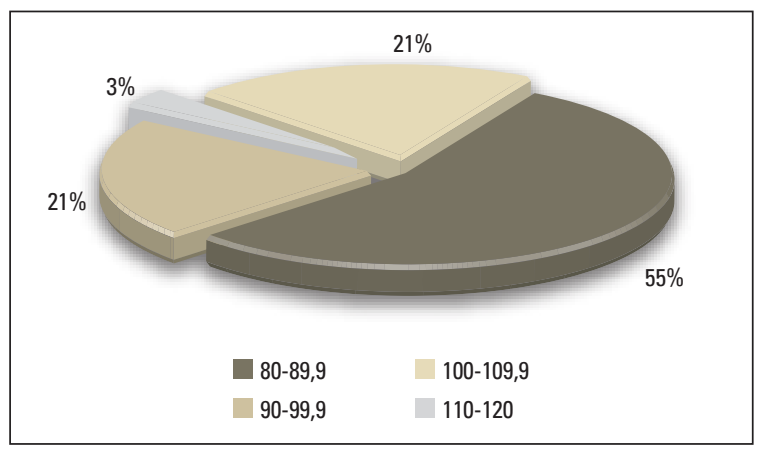

GRÁFICO 3 - Distribuição dos valores de IMPA na telerradiografia. 


\section{DISCUSSÃO}

Durante muitos anos, os ortodontistas basearam seu planejamento ortodôntico em valores normativos encontrados nas análises cefalométri$\operatorname{cas}^{2,4,11,20,21}$, principalmente na definição das inclinações dentárias ideais. Como visto anteriormente, a posição do incisivo inferior era considerada um item primordial e determinante no planejamento ortodôntico ${ }^{11,21}$. Este ideal baseado no posicionamento dos incisivos foi, gradativamente, perdendo a importância, à medida que a meta, teoricamente planejada, era dificilmente atingida na prática ou, quando era, nem sempre resultava em um aspecto facial agradável ou, no mínimo, aceitável.

Além disso, a obtenção do valor exato do IMPA na telerradiografia é uma tarefa das mais difíceis, devido à sobreposição das raízes de todos os dentes anteriores, o que dificulta a confecção do desenho anatômico e a identificação dos pontos cefalométricos com confiabilidade ${ }^{1}$. Com o advento da tomografia computadorizada, a sobreposição dos dentes adjacentes pôde ser eliminada, tornando este exame especialmente útil na aquisição dessas mensurações ${ }^{3}$. Pela utilização de um software para manipulação das imagens tomográficas, pode-se obter um slice que representa fielmente as estruturas necessárias para a obtenção do valor exato de IMPA. Assim sendo, imagens obtidas pela tomografia computadorizada são de grande confiabilida$\mathrm{de}^{\mathrm{I}, 13,15}$, uma vez que tal aparelho produz imagens tridimensionais com a mesma resolução em todos os planos, além de propiciar imagens em três dimensões e possibilitar o isolamento da estrutura anatômica, que é objeto de estudo.

\section{Erro metodológico}

Realizou-se o teste de erro intra-examinador e interexaminadores com o objetivo de aferir a reprodutibilidade e confiabilidade dos valores encontrados. Foram realizadas duas mensurações do valor de IMPA, em 26,31\% da amostra, escolhidos aleatoriamente, pelos dois examinadores. $\mathrm{Na}$ avaliação interexaminadores, os resultados apresen- tados, tanto na telerradiografia $(\mathrm{p}=0,27)$ quanto na tomografia computadorizada $(p=0,155)$, não apresentaram significância estatística $(\mathrm{p}<0,05)$.

Em relação ao erro sistemático intra-examinadores, ambos apresentaram valores de $\mathrm{p}$ não-significantes, ou seja, não houve diferença estatística entre as duas mensurações. Para o erro casual, também não foi encontrado valor representativo de erro significativo para medidas angulares, que, segundo Houston ${ }^{10}$, é de $1,5^{\circ}$. A maior diferença de mensuração encontrada foi de $0,53^{\circ}$.

Assim, com base nesses resultados, pode-se confirmar que ambos os examinadores estavam calibrados para a mensuração do IMPA nos dois métodos e que a nova metodologia apresenta confiabilidade e reprodutibilidade, sugerindo que seja empregada como método de avaliação confiável para esta medida, em pesquisas futuras. Entretanto, novos trabalhos devem ser realizados a fim de confirmar estes dados encontrados e consolidar a utilização de exames complementares digitalizados como um caminho natural para a evolução das ciências e para a pesquisa ortodôntica.

\section{Comparação do valor do IMPA obtido na telerradiografia e na TC}

$\mathrm{Na}$ comparação dos resultados obtidos em cada uma das metodologias deste estudo, observou-se que os resultados variaram bastante. As médias do valor de IMPA apresentadas na TC foram maiores que aquelas apresentadas na telerradiografia $\left(97,93^{\circ}\right.$ e $97,62^{\circ}$, contra $96,03^{\circ}$ e $95,26^{\circ}$, para os primeiro e segundo examinadores, respectivamente). Embora diferente estatisticamente, esta diferença nas médias do IMPA não apresenta significância clínica, uma vez que, ao se fazer o diagnóstico ortodôntico, é mais importante a relação que o incisivo tem com as corticais ósseas, sua inclinação em relação à base óssea de suporte, sua relação com os incisivos superiores e a direção de movimentação planejada no tratamento ortodôntico do que o valor numérico em si.

A tendência do valor mais alto do IMPA na to- 
mografia ocorreu para todos os indivíduos desta amostra, exceto para os indivíduos de número 12 e 14, que apresentaram a tendência contrária, ou seja, os valores na tele se apresentaram maiores que os da TC. Isto ocorreu porque, na telerradiografia, o desenho do incisivo inferior mais vestibularizado foi feito utilizando a raiz do canino que estava vestibularizado. Como conseqüência, o valor do incisivo inferior, que na realidade estava mais verticalizado, sofreu alteração e aumento em seu valor na telerradiografia. A tendência quase absoluta do valor de IMPA ser maior na TC pode ter ocorrido pela diferença no desenho do contorno dos incisivos. Como a TC está livre de sobreposições, o correto desenho do longo eixo do incisivo inferior é possível, permitindo a leitura exata da inclinação do incisivo central mais vestibularizado. Na telerradiografia, há muita sobreposição, particularmente na região dos ápices dos quatro incisivos mais os caninos. Supõese que, no desenho anatômico, o ápice escolhido é o do canino e, uma vez que este se apresentase mais verticalizado, a união à coroa do incisivo gera um desenho cuja medida é menor do que a real. Para os dois indivíduos que apresentaram a tendência oposta, observou-se que os valores de IMPA estavam acima dos $100^{\circ}$, nos dois métodos, incluindo-se também a vestibularização do canino para compensação da má oclusão; na tomografia houve destaque da imagem do incisivo, permitindo que o desenho ficasse mais adequado e real.

A maior diferença encontrada entre os dois métodos foi de mais de $7^{\circ}$, muito significante na elaboração de um diagnóstico ortodôntico, pelos padrões cefalométricos. Neste caso em especial, pode-se supor que a diferença ocorreu porque, na tele, o ápice utilizado foi o do dente canino, dente que, na maioria dos casos, se apresenta verticalizado ou, ainda, com inclinação negativa, fazendo com que a medida diminuísse consideravelmente. $\mathrm{Na}$ TC com a leitura correta da inclinação do incisivo inferior que, nesse indivíduo, apresentava-se bem vestibularizado (como conseqüência da discrepância esquelética maxilomandibular aumentada e pelos mecanismos compensatórios de crescimento e desenvolvimento) - o valor do IMPA aumentou.

$\mathrm{Na}$ análise estatística, encontrou-se diferenças de valores estatisticamente significantes ( $\mathrm{p}<$ $0,05)$ na mensuração dos dois examinadores, com valores de $\mathrm{p}=0,014$ para o examinador 1 e $\mathrm{p}=$ 0,005 para o examinador 2. Estes resultados não surpreendem, já que, no momento da coleta dos dados, observou-se grande dificuldade em escolher na telerradiografia o dente cujo valor de IMPA seria mensurado, em comparação com a TC, que apresentou uma definição impressionante, por não haver qualquer sobreposição de imagem.

Além disso, é importante ressaltar que, na maioria dos casos, o valor do IMPA foi maior na TC. Observando-se os gráficos 2 e 3, é possível verificar que houve aumento da porcentagem dos valores de IMPA da telerradiografia para a TC, apenas naqueles dentes que se apresentavam de $90^{\circ}$ a $99,9^{\circ}$. Isso aconteceu, possivelmente, porque, nessa faixa de valor, a sobreposição dos incisivos com os caninos é mais intensa. Apesar de significante estatisticamente, a variação com aumento de valor para o IMPA, no exame tomográfico, tem pouco significado clínico, quando comparada ao valor encontrado na telerradiografia.

\section{CONCLUSÃO}

Conforme a metodologia empregada e pelos resultados apresentados neste trabalho, pode-se concluir que a obtenção dos valores para o ângulo formado entre o longo eixo do incisivo inferior e o plano mandibular (IMPA) na telerradiografia lateral é um método confiável para uso clínico, porque, embora apresente como regra valores menores que os encontrados em tomografia computadorizada, as diferenças não são significativas clinicamente. Isso era previsível, pela lógica da região analisada e pela limitação que esse método clássico não pode evitar.

Enviado em: setembro de 2006 Revisado e aceito: fevereiro de 2007 


\title{
Computed tomographic evaluation of inferior incisor position
}

\begin{abstract}
Introduction: Orthodontics has always used lateral cephalometric radiographs (LC) as the main examination in the orthodontic diagnosis and treatment plan. The determination of incisor position is part of most cephalometric analysis, including Tweed analysis. However, all radiographic images have limitations, since it is a bidimensional image of a tridimensional structure and overlapping of bone and dental structures often occurs. Computed tomography (CT) allows a trustworthy diagnostic, mainly for its tridimensional images possibility. In this situation it seems reasonable to question the classic cephalometric analysis, creating factors of comparison with computed tomography data. Aim: For this, it was considered the evaluation of the angle between the long axis of the inferior incisor and the mandibular plane (IMPA) in the lateral cephalograph and in the computed tomography. Methods: Nineteen patients, selected for orthodontic treatment, had constituted the sample of this study, 12 female and 7 male, with ages between 16 years and 4 months and 28 years and 2 months. After the collection of data made by two examiners, statistical analyses for the attainment of the errors intra and inter-examiners had been made, using level of significance of $5 \%$. Results and Conclusion: It was concluded that IMPA can be measured in the lateral cephalometric radiographs with trustworthiness, even that its values were smaller when compared with the computed tomography.
\end{abstract}

Key words: Cephalometry. Computed tomography. Orthodontics.

\section{REFERÊNCIAS}

1. BAUMRIND, S.; FRANTZ, R. C. The reliability of cephalometric radiograph measurements. Am. J. Orthod., St. Louis, v. 60, no. 5, p. 111-127, Nov. 1971.

2. BROADBENT, H. B. A new $X$-ray technique and its application to orthodontia. Angle Orthod., Appleton, v. 1, no. 7, p. 45-66, Apr. 1931.

3. CAPELOZZA FILHO, L.; FATTORI, L.; MALTAGLIATI, L. A. Um novo método para avaliar as inclinações dentárias utilizando a tomografia computadorizada. Rev. Dental Press Ortodon. Ortop. Facial, Maringá, v. 10, n. 5, p. 23-29, set./out. 2005.

4. DOWNS, W. Variations in facial relationships: their significance in treatment and prognosis. Am. J. Orthod. Dentofacial Orthop., St. Louis, v. 34, no. 2, p. 812-840, Oct. 1948.

5. FINLAY, L. M. Craniometry and cephalometry: a history prior to the advent of radiography. Angle Orthod., Appleton, v. 50 , no. 4, p. 312-321, Oct. 1980.

6. HAMADA, Y.; KONDOH, T.; NOGUCHI, K.; IINO, M.; ISONO, H.; ISHII, H.; MISHIMA, A.; KOBAYASHI, K.; SETO, K. Application of limited cone beam computed tomography to clinical assessment of alveolar bone grafting: a preliminary report. Cleft Palate Craniofac. J., Pittsburgh, v. 42, no. 2, p. 128-137, Mar. 2005.

7. HANDELMAN, C. S. The anterior alveolus: its importance in limiting orthodontic treatment and its influence on the occurrence of iatrogenic sequelae. Angle Orthod., Appleton, v. 66, no. 2, p. 95-110, 1996.

8. HATCHER, D. C.; ABOUDARA, C. L. Diagnosis goes digital. Am. J. Orthod. Dentofacial Orthop., St. Louis, v. 125, no. 4, p. 512-515, Apr. 2004.

9. HEILAND, M.; SCHULZE, D.; ROTHER, U.; SCHMELZLE, R. Midfacial imaging using digital volume tomography. Int. Congr. Ser., Amsterdam, v. 1256, p. 1230-1234, 2003.

10. HOUSTON, W. J. B. The analysis of errors in orthodontics measurements. Am. J. Orthod. Dentofacial Orthop., St. Louis, v. 83, no. 5, p. 382-390, May 1983.

11. INTERLANDI, S. Linha I na análise morfodiferencial para o diagnóstico ortodôntico. Rev. Fac. Odontol. Univ. São Paulo, São Paulo, v. 9, n. 2, p. 283-310, jul./dez. 1971.

12. LASCALA, C. A.; PANELLA, J.; MARQUES, M. M. Analysis of the accuracy of linear measurements obtained by cone beam computed tomography (CBCT - NewTom). Dentomaxillofac. Radiol., Houndsmills, v. 33, no. 5, p. 291-294, Sept. 2004.
13. MAH, J. K.; DANFORTH, R. A.; BUMANN, A.; HATCHER, D. Radiation absorved in maxillofacial imaging with a new dental computed tomography device. Oral Surg. Oral Med. Oral Pathol. Oral Radiol. Endod., St. Louis, v. 96, no. 4, p. 508-513, Oct. 2003.

14. MARGOLIS, H. I. The axial inclination of the mandibular incisor. Am. J. Orthod. Dentofacial Orthop., St. Louis, v. 29, no. 10, p. 571-594, Oct. 1943

15. MARMULLA, R.; WÖRTCHE, R.; MÜHLING, J.; HASSFELD, S. Geometric accuracy of the NewTom 9000 Cone Beam CT. Dentomaxillofac. Radiol., Houndsmills, v. 34, no. 1, p. 28-31, Jan. 2005.

16. MARTINS, D. R. Estudo comparativo dos valores cefalométricos das análises da Downs e Tweed, com adolescentes brasileiros leucodermas de origem mediterrânea. Ortodontia, São Paulo, v. 14, n. 2, p. 101-116, maio 1981.

17. RUSTMEYER, P.; STREUBÜHR, U.; SUTTMOELLER, J. LOWdose dental computed tomography: significant dose reduction without loss of image quality. Acta Radiol., Stockholm, v. 45, no. 8, p. 847-853, Dec. 2004.

18. SCHULZE, D.; HEILAND, M.; SCHMELZLE, R.; ROTHER, U. J. Diagnostic possibilities of cone-beam computed tomography in the facial skeleton. Int. Congr. Ser., Amsterdam, v. 1268, p. 1179-1183, 2004.

19. SCHULZE, D.; HEILAND, M.; THURMANN, H.; ADAM, G. Radiation exposure during midfacial imaging using 4- and 16-slice computed tomography, cone beam computed tomography systems and conventional radiography Dentomaxillofac. Radiol., Houndsmills, v. 33, no. 2, p. 83-86, Mar. 2004.

20. STEINER, C. C. The use of cephalometrics as an aid to planning and assessing orthodontic treatment. Am. J. Orthod. Dentofacial Orthop., St. Louis, v. 46, no. 10, p. 721-735, Oct. 1960.

21. TWEED, C. H. The Frankfort-mandibular incisor Angle (FMIA) in orthodontic diagnosis, treatment planning, and prognosis. Angle Orthod., Appleton, v. 24, no. 3, p. 121-169, July 1954.

Endereço para correspondência

Liana Fattori

Rua Primeiro de Maio, 188 cj.111 - Centro

CEP: 09.015-030 - Santo André/SP

E-mail: dralianafattori@uol.com.br 